\title{
On the space-time curvature experienced by quasiparticle excitations in the Painlevé-Gullstrand effective geometry
}

\author{
Uwe R. Fischer \\ Department of Physics, University of Illinois at Urbana-Champaign \\ 1110 West Green Street, Urbana, Illinois 61801-3080 \\ Matt Visser \\ School of Mathematical and Computing Sciences \\ Victoria University of Wellington \\ P.O. Box 600, Wellington, New Zealand
}

\begin{abstract}
We consider quasiparticle propagation in constant-speed-of-sound (iso-tachic) and almost incompressible (iso-pycnal) hydrodynamic flows, using the technical machinery of general relativity to investigate the "effective space-time geometry" that is probed by the quasiparticles. This effective geometry, described for the quasiparticles of condensed matter systems by the Painlevé-Gullstrand metric, generally exhibits curvature (in the sense of Riemann), and many features of quasiparticle propagation can be re-phrased in terms of null geodesics, Killing vectors, and Jacobi fields. As particular examples of hydrodynamic flow we consider shear flow, a constant-circulation vortex, flow past an impenetrable cylinder, and rigid rotation.

PACS numbers: 02.40.Ky
\end{abstract}

\section{INTRODUCTION}

The description of many natural phenomena is most vividly carried out in terms of hydrodynamics, because the concept of a streaming liquid elucidates and helps to understand the physical significance and structure of an underlying theory [1]. In its classical sense 2, 3], hydrodynamics describes the motion of a continuum, characterized by a velocity and density distribution, which for a perfect fluid and in the nonrelativistic limit is described by the Euler and continuity equations. It has been recognized about twenty years ago by Unruh [4], that the propagation of small perturbations on such a hydrodynamic background, which is itself governed by a continuum version of Newtonian physics, may be cast into the form of a "relativistic" scalar wave equation

$$
\Phi \equiv \frac{1}{\sqrt{-g}} \partial_{\mu}\left(\sqrt{-g} g^{\mu \nu} \partial_{\nu} \Phi\right)=0
$$

for the velocity potential $\Phi$ of the perturbations. The disturbances propagate in an effective space-time with metric $g_{\mu \nu}$, which is in general curved. The metric $g_{\mu \nu}$ was later on shown to be of the Painlevé-Gullstrand form [5], originally invented as an alternative to the Schwarzschild form of the solution of the Einstein equations for a point mass source. With the advent of effective curved spacetime theories, it became apparent that the PainlevéGullstrand representation of the metric appears in a host of such theories. They comprise, besides the conventional Euler fluid [4, 6], superfluid ${ }^{3} \mathrm{He}-\mathrm{A}$ [7, 8], atomic Bosecondensed vapors 9, 10], and general dielectric (quantum) matter [11, 12, 13].

An interesting and important feature of the PainlevéGullstrand metric is that it continues to give an appropriate physical description for quasiparticle propaga- tion even when the effective space-time possesses a horizon [14]. This occurs because the condensed matter origin of the metric in the Painlevé-Gullstrand form is the spectrum of elementary excitations (quasiparticles) [15], which is primary. This physical energy spectrum, from which the metric is obtained using the fact that for massless quasiparticles the energy spectrum is

$$
g^{\mu \nu} p_{\mu} p_{\nu}=0,
$$

which must be well-defined and, in particular, real everywhere in the system. In contrast, for the Schwarzschild form of the metric the spectrum reads

$$
E^{2}=c^{2}\left(1-\frac{r_{\mathrm{S}}}{r}\right)^{2} p_{r}^{2}+c^{2}\left(1-\frac{r_{\mathrm{S}}}{r}\right) p_{\perp}^{2},
$$

where $r_{\mathrm{S}}$ is the usual Schwarzschild radius and $p_{r}, p_{\perp}$ are radial and transverse components of the quasiparticle momentum, respectively. The velocity $c$ plays the role of the speed of light and is equal to the sound speed for phonons. This "Schwarzschild form" of the spectrum exhibits imaginary mode frequencies and consequently leads to instability of the condensed matter system if a horizon is present, because it has sections of the transverse momentum $p_{\perp}$ which result in $E^{2}<0$ inside the horizon. The Painlevé-Gullstrand metric, on the other hand, gives real frequencies throughout a condensed matter system possessing a quasiparticle horizon, which can thus be stable.

The non-equivalence of Schwarzschild and PainlevéGullstrand form of the metric is related to the fact that the coordinate transformation relating the Schwarzschild solution and the Painlevé-Gullstrand representation becomes singular at the horizon [14]. This fact has, inter alia, led to the usage of Painlevé-Gullstrand co-ordinates for investigations of Hawking radiation in the "conventional" black hole context of gravitational theory [16, 17], 
because these co-ordinates are nonsingular through the horizon, making the appropriate vacuum definition there much simpler.

The intrinsic characteristics of a curved space-time are described in a covariant way by the Riemann tensor [18, 19]. Our objective in this paper is to describe the Riemannian curvature of the effective spaces described by the Painlevé-Gullstrand metric, in the underlying hydrodynamic terms appropriate to a flowing background fluid. We shall focus on two physical situations: quasiparticles in flows with a constant speed of sound (isotachic flows), and quasiparticles in an almost incompressible (iso-pycnal) hydrodynamic flow. By "almost incompressible" we mean that we take both the background density and the quasiparticle propagation speed relative to the medium to be constants, and concentrate on those effects that are due to motion of the medium, i.e., its velocity distribution. In other words, even if a fluid has a constant "refractive index", focussing and defocussing effects can be engendered through motion of the fluid.

As particularly interesting examples we demonstrate how the tracks of quasiparticles are distorted by propagation through a shear flow, a constant-circulation vortex flow, around an impenetrable cylinder, and how they propagate through a rigidly rotating fluid. In a more general context we provide a local definition of "focal length" in terms of the Riemann tensor, and show how the affine and "natural" (using the Newtonian background time) parameterizations of null geodesics can be related to each other.

\section{PAINLEVÉ-GULLSTRAND CURVATURE IN $3+1$ DIMENSIONS}

In the following discussion the quasiparticle spectrum is assumed to be linear in the fluid rest frame for "small" quasiparticle momenta, $E=c|\boldsymbol{p}|$ corresponding to (2), and deviating from linearity for momenta approaching the "Planck scale" of the system at hand. In general the (3+1)-dimensional Painlevé-Gullstrand metric [5] reads

$$
g_{t t}=-\frac{\rho}{c}\left[c^{2}-\boldsymbol{v}^{2}\right], \quad g_{t i}=-\frac{\rho}{c} v_{i}, \quad g_{i j}=\frac{\rho}{c} \delta_{i j} .
$$

That is, the metric has space-time interval

$$
\mathrm{d} s^{2}=\frac{\rho}{c}\left[-c^{2} \mathrm{~d} t^{2}+\delta_{i j}\left(\mathrm{~d} x^{i}-v^{i} \mathrm{~d} t\right)\left(\mathrm{d} x^{j}-v^{j} \mathrm{~d} t\right)\right] .
$$

By special convention, the indices on the 3 -velocity are always raised and lowered using the flat 3-dimensional Cartesian metric so that $v_{i}=v^{i}$.

In the case of irrotational fluid flow (for instance in a superfluid outside the cores of the (singular) quantized vortices), the d'Alembertian equation (11) can be derived directly from a linearization procedure based on the Euler and continuity equations [4, [6] ; the existence and relevance of the Painlevé-Gullstrand effective metric then follows as a rigorous theorem. If distributed vorticity is present, the situation is more subtle [20]: In hydrodynamics with distributed vorticity one obtains a rather complicated system of coupled differential equations, one of which contains the d'Alembertian operator (and therefore also contains the effective metric) as a subsidiary quantity [20]. Thus for hydrodynamics with distributed vorticity, the effective metric is not the whole story but certainly an important part of the story. In particular, if one appeals to the eikonal approximation (in this context identical to the WKB approximation) one can derive Pierce's approximate wave equation 21]. In this approximation one can write down the quasiparticle spectrum directly in terms of the effective metric [20].

Note that the constant-time hypersurfaces are conformal to ordinary flat Cartesian space. As long as we are interested in quasiparticles that propagate along the null cones of this effective metric (that is, quasiparticles moving at the speed $c$ relative to the medium), it is permissible to neglect the overall conformal factor of $\rho / c$ and consider the simplified metric

$$
g_{t t}=-\left[c^{2}-\boldsymbol{v}^{2}\right], \quad g_{t i}=-v_{i}, \quad g_{i j}=\delta_{i j} .
$$

(This is simply the statement that conformal transformations leave null curves and, in particular, null geodesics, invariant.) The inverse of this simplified metric is

$$
g^{t t}=-\frac{1}{c^{2}}, \quad g^{t i}=-\frac{v^{i}}{c^{2}}, \quad g^{i j}=\delta^{i j}-\frac{v^{i} v^{j}}{c^{2}} .
$$

Note that the Newtonian time parameter $t$ provides a preferred foliation of the spacetime into space+time, and that this preferred foliation will prove very useful.

Suppose now that the speed of sound is iso-tachic, independent of position and time. Then we can choose coordinates to set the speed $c$ of linear quasiparticle dispersion equal to unity, a convention adopted in the formulae below. The $(3+1)$-dimensional Painlevé-Gullstrand metric [5] then reads

$$
g_{t t}=-1+\boldsymbol{v}^{2}, \quad g_{t i}=-v_{i}, \quad g_{i j}=\delta_{i j} .
$$

In general relativistic language the lapse function in the ADM formulation [19] is now unity and all the spacetime curvature is encoded in the shift function - which here describes the physical velocity of the fluid. The inverse metric is

$$
g^{t t}=-1, \quad g^{t i}=-v^{i}, \quad g^{i j}=\delta^{i j}-v^{i} v^{j} .
$$

Turning to the computation of curvature, the 24 independent connection coefficients read (cf. [22])

$$
\begin{aligned}
\Gamma^{t}{ }_{i j} & =D_{i j}, \\
\Gamma^{t}{ }_{t t} & =v_{i} v_{k} D_{i k}=\frac{1}{2}(\boldsymbol{v} \cdot \nabla) \boldsymbol{v}^{2}, \\
\Gamma^{t}{ }_{t i} & =-v_{j} D_{i j}, \\
\Gamma^{i}{ }_{j k} & =v_{i} D_{j k}, \\
\Gamma^{i}{ }_{t t} & =-\partial_{t} v_{i}-v_{k} \partial_{i} v_{k}+v_{i} v_{l} v_{k} D_{l k}, \\
& =-\partial_{t} v_{i}-\frac{1}{2}\left(\delta^{i j}-v^{i} v^{j}\right) \partial_{j} \boldsymbol{v}^{2}, \\
\Gamma^{i}{ }_{t j} & =-v_{i} v_{k} D_{j k}+\Omega_{i j} .
\end{aligned}
$$


Here we have defined the deformation rate and angular velocity tensors by

$$
\begin{aligned}
D_{i j} & =\frac{1}{2}\left(\partial_{i} v_{j}+\partial_{j} v_{i}\right) \\
& \left.=\partial_{(i} v_{j}\right)=D_{j i}, \\
\operatorname{Tr} \boldsymbol{D} & =\operatorname{div} \boldsymbol{v}, \\
\Omega_{i j} & =\frac{1}{2}\left(\partial_{i} v_{j}-\partial_{j} v_{i}\right) \\
& =\partial_{[i} v_{j]}=-\Omega_{j i} .
\end{aligned}
$$

The deformation rate is in general relativistic language the extrinsic curvature of the constant-time hypersurfaces, while the angular velocity tensor is in fluid mechanics language equivalent to the vorticity vector defined via $\omega^{i}=\epsilon^{i j k} \Omega_{j k}$. The above tensors result in the unique decomposition of $\partial_{i} v_{j}=(\nabla \otimes \boldsymbol{v})_{i j}=D_{i j}+\Omega_{i j}$ into a symmetric and an antisymmetric tensor.

The components of the Riemann curvature tensor afford the basic symmetries $R_{[\mu \nu][\rho \lambda]}=R_{[\rho \lambda][\mu \nu]}$, which are supplemented by $R_{[\mu \nu \rho \lambda]}=0$ and $R_{\mu[\nu \rho \lambda]}=0$ [19]. The Riemann components that need to be calculated are thus $R_{t i t j}, R_{i j k l}$, and $R_{t i j k}$, the rest follow by the (anti-)symmetry properties. A tedious but straightforward computation (which follows a variant of the GaussCodazzi decomposition) yields

$$
\begin{aligned}
R_{i j k l}= & D_{i k} D_{j l}-D_{i l} D_{j k} \\
R_{t i j k}= & -\partial_{i} \Omega_{j k}+v_{l}\left(D_{k l} D_{i j}-D_{j l} D_{i k}\right) \\
R_{t i t j}= & -\partial_{t} D_{i j}+(\boldsymbol{D} \boldsymbol{\Omega}+\boldsymbol{\Omega} \boldsymbol{D})_{i j}-\left(\boldsymbol{D}^{2}\right)_{i j} \\
& -v_{k} v_{k, i j}+v_{k} v_{l}\left(D_{k l} D_{i j}-D_{j k} D_{i l}\right) .
\end{aligned}
$$

Here we have defined $(\boldsymbol{D} \boldsymbol{\Omega}+\boldsymbol{\Omega} \boldsymbol{D})_{i j} \equiv D_{i k} \Omega_{k j}+\Omega_{i k} D_{k j}$, and similarly $\left(\boldsymbol{D}^{2}\right)_{i j} \equiv D_{i k} D_{k j}$.

The appearance and interpretation of the Riemann components may be greatly simplified if we consider them in an orthonormal, locally Minkowskian tetrad frame $\left\{e^{a}{ }_{\mu}\right\}$. Greek indices denote the usual spacetime indices, Roman letters from the beginning of the alphabet indicate tetrad indices, while Roman letters from the middle of the alphabet denote space indices. Whenever there is any chance of confusion, carets on indices are used to indicate that the components are given in the tetrad frame. The tetrad frame $\left\{e^{a}{ }_{\mu}\right\}$ is defined by

$$
g_{\mu \nu}=\eta_{a b} e_{\mu}^{a} e_{\nu}^{b}
$$

In the simplest gauge it is given by

$$
\begin{aligned}
e^{\hat{t}_{t}} & =1, \quad e_{i}^{\hat{t}}=0, \\
e^{\hat{\imath}}{ }_{t} & =-v^{i}, \quad e^{\hat{\jmath}}{ }_{i}=\delta^{\hat{\jmath}_{i}} .
\end{aligned}
$$

The inverse basis satisfies

$$
g^{\mu \nu}=\eta^{a b} e_{a}^{\mu} e_{b}^{\nu} .
$$

Note the use of index placement to distinguish $e^{a}{ }_{\mu}$ from its inverse $e_{a}{ }^{\mu}$. Hence $e^{a}{ }_{\mu} e_{b}{ }^{\mu}=\delta^{a}{ }_{b}$ as well as $e_{a}{ }^{\mu} e^{a}{ }_{\nu}=$ $\delta^{\mu}{ }_{\nu}$. In a time plus space decomposition

$$
\begin{aligned}
& e_{\hat{t}}^{t}=1, \quad e_{\hat{\imath}}^{t}=0, \\
& e_{\hat{t}}{ }^{i}=v^{i}, \quad e_{\hat{\imath}}^{j}=\delta_{\hat{\imath}}^{j} .
\end{aligned}
$$

Thus, for any given vector with components $X^{\mu}$ the components in the various frames are related by

$$
X_{a} \equiv e_{a}{ }^{\mu} X_{\mu} \equiv\left(X_{\hat{t}} ; X_{\hat{\imath}}\right)=\left(X_{t}+v^{j} X_{j} ; X_{i}\right)
$$

and

$$
X^{a} \equiv e^{a}{ }_{\mu} X^{\mu} \equiv\left(X^{\hat{t}} ; X^{\hat{\imath}}\right)=\left(X^{t} ; X^{i}-v^{i} X^{t}\right) .
$$

These index conventions greatly simplify the formulae below. Calculating the Riemann tensor in the tetrad frame gives

$$
\begin{aligned}
& R_{\hat{\imath} \hat{\jmath} \hat{k} \hat{l}}=D_{i k} D_{j l}-D_{i l} D_{j k}, \\
& R_{\hat{t} \hat{\imath} \hat{\jmath} \hat{k}}=-\partial_{i} \Omega_{j k}, \\
& R_{\hat{t} \hat{\imath} \hat{\imath} \hat{\jmath}}=-\frac{\mathrm{d}}{\mathrm{d} t} D_{i j}-\left(\boldsymbol{D}^{2}\right)_{i j}+(\boldsymbol{D} \boldsymbol{\Omega}+\boldsymbol{\Omega} \boldsymbol{D})_{i j},
\end{aligned}
$$

where

$$
\frac{\mathrm{d}}{\mathrm{d} t}=\partial_{t}+\boldsymbol{v} \cdot \nabla
$$

is the usual convective derivative. The tetrad components $R_{a b c d}$ tell us how a Lagrangian observer moving with the fluid perceives the curvature of the effective space-time described by the Painlevé-Gullstrand metric (8).

The components in the tetrad and co-ordinate frames are related by

$$
R_{\alpha \beta \gamma \delta}=e_{\alpha}^{a} e_{\beta}^{b} e_{\gamma}^{c} e_{\delta}^{d} R_{a b c d} .
$$

In the tetrad frame, the Ricci tensor

$$
R_{a b}=R_{a c b}^{c}=-R_{\hat{t} a \hat{t} b}+R_{\hat{k} a \hat{k} b}
$$

has the components

$$
\begin{aligned}
R_{\hat{t} \hat{t}} & =R_{\hat{k} \hat{t} \hat{k} \hat{t}}=R_{\hat{t} \hat{k} \hat{t} \hat{k}} \\
& =-\frac{\mathrm{d}}{\mathrm{d} t} \operatorname{Tr} \boldsymbol{D}-\operatorname{Tr}\left(\boldsymbol{D}^{2}\right) \\
R_{\hat{t} \hat{\imath}} & =-R_{\hat{t} \hat{k} \hat{k} \hat{\imath}} \\
& =\partial_{k} \Omega_{k i}=\frac{1}{2} \Delta v_{i}-\frac{1}{2} \partial_{i}(\operatorname{Tr} \boldsymbol{D}) \\
& =-\frac{1}{2}(\nabla \times \boldsymbol{\omega})_{i}, \\
R_{\hat{\imath} \hat{\jmath}} & =-R_{\hat{t} \hat{\imath} \hat{\jmath} \hat{\jmath}}+R_{\hat{k} \hat{\imath} \hat{k} \hat{\jmath}} \\
& =\frac{\mathrm{d}}{\mathrm{d} t} D_{i j}-(\boldsymbol{D} \boldsymbol{\Omega}+\boldsymbol{\Omega} \boldsymbol{D})_{i j}+D_{i j} \operatorname{Tr} \boldsymbol{D},
\end{aligned}
$$

where we remind the reader that we have defined the vorticity vector

$$
\omega_{i}=\omega^{i}=\epsilon^{i j k} \Omega_{j k}=(\operatorname{rot} \boldsymbol{v})_{i}=(\nabla \times \boldsymbol{v})_{i} .
$$


The curvature scalar thus becomes

$$
\begin{aligned}
R & =R_{a b} \eta^{a b}=-R_{\hat{t} \hat{t}}+R_{\hat{k} \hat{k}} \\
& =2 \frac{\mathrm{d}}{\mathrm{d} t} \operatorname{Tr} \boldsymbol{D}+(\operatorname{Tr} \boldsymbol{D})^{2}+\operatorname{Tr}\left(\boldsymbol{D}^{2}\right),
\end{aligned}
$$

and contains the trace of the deformation tensor and the trace of its square, but not the vorticity. Finally, the Einstein tensor takes the form

$$
\begin{aligned}
G_{\hat{t} \hat{t}}= & R_{\hat{t} \hat{t}}+\frac{1}{2} R \\
= & \frac{1}{2}(\operatorname{Tr} \boldsymbol{D})^{2}-\frac{1}{2} \operatorname{Tr}\left(\boldsymbol{D}^{2}\right) \\
G_{\hat{t} \hat{\imath}}= & R_{\hat{t} \hat{\imath}}=-\frac{1}{2}(\nabla \times \boldsymbol{\omega})_{i} \\
G_{\hat{\imath} \hat{\jmath}}= & R_{\hat{\imath} \hat{\jmath}}-\frac{1}{2} \delta_{\hat{\imath} \hat{\jmath}} R \\
= & \frac{\mathrm{d}}{\mathrm{d} t}\left(D_{i j}-\delta_{i j} \operatorname{Tr} \boldsymbol{D}\right)+\operatorname{Tr} \boldsymbol{D}\left(D_{i j}-\frac{1}{2} \delta_{i j} \operatorname{Tr} \boldsymbol{D}\right) \\
& -\frac{1}{2} \delta_{i j} \operatorname{Tr}\left(\boldsymbol{D}^{2}\right)-(\boldsymbol{D} \boldsymbol{\Omega}+\boldsymbol{\Omega} \boldsymbol{D})_{i j}
\end{aligned}
$$

We emphasise that although the Ricci and Einstein tensors are nontrivial, and certainly objects of physical interest, there is at this level no need for or justification for imposing Einstein equations - though these Ricci and Einstein tensors are properties of the flow, they are not directly related to the stress-energy tensor generating that flow and thus the effective space-time curvature experienced by the quasiparticles. In superfluids, for example, the "Einstein action" proportional to the curvature scalar (31) is smaller than the simple kinetic energy of the superflow by the factor $a^{2} / l^{2}$, where $a$ is the atomic scale and $l$ the scale on which the velocity field varies [7], so that the "Einstein action" is subdominant in determining the velocity field.

It is sometimes convenient to work with the conformally invariant, traceless part of curvature. This is given by the Weyl tensor [23]:

$$
C_{a b c d}=R_{a b c d}+\eta_{a[d} R_{c] b}+\eta_{b[c} R_{d] a}+\frac{1}{3} R \eta_{a[c} \eta_{d] b},
$$

where the brackets indicate anti-symmetrization on the indices they enclose. This gives

$$
\begin{aligned}
C_{\hat{\imath} \hat{\jmath} \hat{k} \hat{l}}= & R_{\hat{\imath} \hat{\jmath} \hat{k} \hat{l}}+\delta_{\hat{\imath}[\hat{l}} R_{\hat{k}] \hat{\jmath}}+\delta_{\hat{\jmath}[\hat{k}} R_{\hat{l}] \hat{\imath}}+\frac{1}{3} R \delta_{\hat{\imath}[\hat{k}} \delta_{\hat{l}] \hat{\jmath}}, \\
C_{\hat{t} \hat{\imath} \hat{\jmath} \hat{k}}= & -\partial_{i} \Omega_{j k}-\frac{1}{2} \delta_{i[j}(\nabla \times \boldsymbol{\omega})_{k]}, \\
C_{\hat{t} \hat{\imath} \hat{t} \hat{\jmath}}= & -\frac{1}{2} \frac{\mathrm{d}}{\mathrm{d} t}\left(D_{i j}-\frac{1}{3} \delta_{i j} \operatorname{Tr}(\boldsymbol{D})\right) \\
& -\left(\boldsymbol{D}^{2}\right)_{i j}+\frac{1}{3} \delta_{i j} \operatorname{Tr}\left(\boldsymbol{D}^{2}\right) \\
& +\frac{1}{2} \operatorname{Tr}(\boldsymbol{D})\left(D_{i j}-\frac{1}{3} \delta_{i j}(\operatorname{Tr} \boldsymbol{D})\right) \\
& +\frac{1}{2}(\boldsymbol{D} \boldsymbol{\Omega}+\boldsymbol{\Omega} \boldsymbol{D})_{i j} .
\end{aligned}
$$

\section{EXAMPLES}

\section{A. General iso-pycnal flows}

Suppose now that the flow is not only iso-tachic (constant speed of sound) but also iso-pycnal (constant background density). This corresponds to an "almost incompressible" fluid such as water. The major change from the previous section is the simplification that comes from the continuity equation:

$$
\frac{\mathrm{d} \rho}{\mathrm{d} t}=0 \quad \Longrightarrow \quad \nabla \cdot \boldsymbol{v}=0 \quad \Longrightarrow \quad \operatorname{Tr} \boldsymbol{D}=0 .
$$

The form of the Riemann tensor is not affected, though for the Ricci tensor we now have

$$
\begin{aligned}
R_{\hat{t} \hat{t}} & =-\operatorname{Tr}\left(\boldsymbol{D}^{2}\right) \\
R_{\hat{t} \hat{\imath}} & =\frac{1}{2} \Delta v_{i} \\
R_{\hat{\imath} \hat{\jmath}} & =\frac{\mathrm{d}}{\mathrm{d} t} D_{i j}-(\boldsymbol{D} \boldsymbol{\Omega}+\boldsymbol{\Omega} \boldsymbol{D})_{i j}
\end{aligned}
$$

The Ricci scalar simplifies to

$$
R=\operatorname{Tr}\left(\boldsymbol{D}^{2}\right)
$$

Thus the Ricci curvature scalar is positive semidefinite for iso-pycnal flows, and vanishes if and only if the deformation $\boldsymbol{D}$ is zero.

The Einstein tensor is now

$$
\begin{aligned}
G_{\hat{t} \hat{t}} & =-\frac{1}{2} \operatorname{Tr}\left(\boldsymbol{D}^{2}\right) \\
G_{\hat{t} \hat{\imath}} & =\frac{1}{2} \Delta v_{i}, \\
G_{\hat{\imath} \hat{\jmath}} & =\frac{\mathrm{d}}{\mathrm{d} t} D_{i j}-\frac{1}{2} \delta_{i j} \operatorname{Tr}\left(\boldsymbol{D}^{2}\right)-(\boldsymbol{D} \boldsymbol{\Omega}+\boldsymbol{\Omega} \boldsymbol{D})_{i j} .
\end{aligned}
$$

Finally the Weyl tensor for iso-pycnal flows reduces to

$$
\begin{aligned}
C_{\hat{\imath} \hat{\jmath} \hat{k} \hat{l}}= & R_{\hat{\imath} \hat{\jmath} \hat{k} \hat{l}}+\delta_{\hat{\imath}[\hat{l}} R_{\hat{k}] \hat{\jmath}}+\delta_{\hat{\jmath}[\hat{k}} R_{\hat{l}] \hat{\imath}}+\frac{1}{3} R \delta_{\hat{\imath}[\hat{k}} \delta_{\hat{l}] \hat{\jmath}}, \\
C_{\hat{t} \hat{\imath} \hat{\jmath} \hat{k}}= & \left.-\partial_{i} \Omega_{j k}+\delta_{i[j} \Delta v_{k}\right] \\
C_{\hat{t} \hat{\imath} \hat{t} \hat{\jmath}}= & -\frac{1}{2} \frac{\mathrm{d}}{\mathrm{d} t} D_{i j}+\frac{1}{2}(\boldsymbol{D} \boldsymbol{\Omega}+\boldsymbol{\Omega} \boldsymbol{D})_{i j} \\
& -\left(\boldsymbol{D}^{2}\right)_{i j}+\frac{1}{3} \delta_{i j} \operatorname{Tr}\left(\boldsymbol{D}^{2}\right) .
\end{aligned}
$$

\section{B. Shear flow}

As a first simple example of a nontrivial incompressible flow ( $\operatorname{Tr} \boldsymbol{D}=0$ ), consider the flow with constant shear

$$
\boldsymbol{v}=\omega_{0}(0, x, 0)
$$

which has both constant deformation $D_{x y}=D_{y x}=\frac{1}{2} \omega_{0}$ and constant vorticity $\omega_{z}=\omega_{0}=2 \Omega_{x y}=-2 \Omega_{y x}$ (all 
other components vanishing) [24]. The Riemann curvature components are

$$
\begin{aligned}
& R_{\hat{t} \hat{\imath} \hat{t} \hat{\jmath}}=-\frac{1}{4} \omega_{0}^{2} \mathcal{P}_{i j}, \\
& R_{\hat{t} \hat{\imath} \hat{\jmath} \hat{k}}=0 \\
& R_{\hat{\imath} \hat{\jmath} \hat{k} \hat{l}}=\frac{1}{4} \omega_{0}^{2}\left(\theta_{i k} \theta_{j l}-\theta_{i l} \theta_{j k}\right),
\end{aligned}
$$

where $\theta_{i k}=\theta_{k i}$ is unity if $(i k)=(x y)$ and zero otherwise. The projection operator

$$
\mathcal{P}_{i j} \equiv \delta_{i j}-n_{i} n_{j}
$$

where $\boldsymbol{n}=(0,0,1)$ is a unit vector in $z$ direction ensures that the curvature has nonzero components only in the $x$ and $y$ directions.

For the Ricci and Einstein tensors

$$
\begin{aligned}
R_{\hat{t} \hat{\imath}} & =R_{\hat{\imath} \hat{\jmath}}=0, \\
R_{\hat{t} \hat{t}} & =-\frac{1}{2} \omega_{0}^{2}=\operatorname{Tr}\left(\boldsymbol{D}^{2}\right), \\
R & =\frac{1}{2} \omega_{0}^{2}, \\
G_{\hat{t} \hat{t}} & =-\frac{1}{4} \omega_{0}^{2}, \\
G_{\hat{\imath} \hat{\jmath}} & =-\frac{1}{4} \omega_{0}^{2} \delta_{i j}, \\
G_{\hat{t} \hat{\imath}} & =0 .
\end{aligned}
$$

Thus the quasiparticles are seen in their effective spacetime to be moving on a $(3+1)$-dimensional manifold of constant scalar curvature, with radius of curvature inversely proportional to the shearing rate $\omega_{0}$.

\section{Vortex flow of constant circulation}

A somewhat more interesting case is the constantcirculation flow in the $x-y$ plane

$$
v_{y}=\frac{\gamma x}{x^{2}+y^{2}}, \quad v_{x}=-\frac{\gamma y}{x^{2}+y^{2}}
$$

appropriate to a vortex flow well outside the central core, where the circulation is $\oint \boldsymbol{v} \cdot \boldsymbol{d} s=2 \pi \gamma$. In this case you would not want to trust the geometry for $r<r_{c}=\gamma$ because at $r=r_{c}$ the flow goes supersonic. This flow has

$$
\begin{aligned}
D_{x x} & =\frac{2 \gamma x y}{r^{4}}=-D_{y y} \\
D_{x y} & =\frac{\gamma\left(y^{2}-x^{2}\right)}{r^{4}}=D_{y x} \\
D_{i z} & =D_{z i}=0 \\
\Omega_{i j} & =0
\end{aligned}
$$

Note the "duality" between the vortex core and the far field. In the core the deformation rate is zero and the vorticity is non-zero, while in the far field it is the vorticity that is zero and deformation that is non-zero. The Riemann curvature tensor takes the form:

$$
\begin{aligned}
R_{\hat{x} \hat{y} \hat{x} \hat{y}} & =\operatorname{det} \boldsymbol{D}=-\frac{\gamma^{2}}{r^{4}}, \\
R_{\hat{t} \hat{\imath} \hat{\jmath} \hat{k}} & =0, \\
R_{\hat{t} \hat{\imath} \hat{t} \hat{\jmath}} & =-(\boldsymbol{v} \cdot \nabla) D_{i j}-\left(\boldsymbol{D}^{2}\right)_{i j} \\
& =-(\boldsymbol{v} \cdot \nabla) D_{i j}-\frac{\gamma^{2}}{r^{4}} \mathcal{P}_{i j} .
\end{aligned}
$$

More explicitly

$$
\begin{aligned}
R_{\hat{t} \hat{x} \hat{t} \hat{x}} & =\frac{\gamma^{2}}{r^{6}}\left(y^{2}-3 x^{2}\right), \\
R_{\hat{t} \hat{y} \hat{t} \hat{y}} & =\frac{\gamma^{2}}{r^{6}}\left(x^{2}-3 y^{2}\right), \\
R_{\hat{t} \hat{x} \hat{t} \hat{y}} & =-\frac{4 \gamma^{2} x y}{r^{6}}, \\
R_{\hat{t} \hat{t} \hat{\jmath}} & =-\frac{\gamma^{2}}{r^{6}}\left(4 x_{i} x_{j}-\delta_{i j} r^{2}\right) .
\end{aligned}
$$

Therefore the Ricci tensor, curvature scalar, and Einstein tensor read

$$
\begin{aligned}
R_{\hat{t} \hat{t}} & =-\frac{2 \gamma^{2}}{r^{4}}, \quad R_{\hat{t} \hat{\imath}}=R_{\hat{\imath} \hat{\jmath}}=0, \\
R & =\frac{2 \gamma^{2}}{r^{4}} \\
G_{\hat{t} \hat{t}} & =-\frac{\gamma^{2}}{r^{4}}, \quad G_{\hat{\imath} \hat{\jmath}}=-\delta_{i j} \frac{\gamma^{2}}{r^{4}}, \\
G_{\hat{t} \hat{\imath}} & =0 .
\end{aligned}
$$

It is mildly amusing to note that the vortex geometry is uniquely determined by the cylindrical symmetry plus the equation $G_{a b} \propto \delta_{a b}\left(\right.$ not $\left.\eta_{a b}\right)$.

\section{Streaming motion past a cylinder}

The most complex flow we discuss here is provided by the two-dimensional streaming motion from right to left past a cylinder of radius $a$. According to the circle theorem [3], the complex velocity potential of such a flow is given by

$$
w=U\left(Z+\frac{a^{2}}{Z}\right)
$$

where $Z=x+i y$ and $U$ is the velocity at infinity in negative $x$ direction. This results in the flow

$$
v_{x}=-U\left(1+a^{2} \frac{y^{2}-x^{2}}{r^{4}}\right), \quad v_{y}=2 U x y \frac{a^{2}}{r^{4}} .
$$

The velocity at infinity is restricted to be $U<1 / 2$, for the maximal velocity on the cylinder surface to be less 
than the speed of sound. The formulae for deformation and vorticity (which is identically zero for this flow) read

$$
\begin{aligned}
D_{x x} & =\frac{2 U a^{2}}{r^{6}} x\left(3 y^{2}-x^{2}\right)=-D_{y y} \\
D_{x y} & =\frac{2 U a^{2}}{r^{6}} y\left(y^{2}-3 x^{2}\right)=D_{y x} \\
D_{i z} & =D_{z i}=0 \\
\Omega_{i j} & =0 .
\end{aligned}
$$

The Riemann components show that the flow past a cylinder, due to its reduced symmetry, yields a more complicated space-time geometry for quasiparticles than the vortex flow:

$$
\begin{aligned}
R_{\hat{x} \hat{y} \hat{x} \hat{y}} & =\operatorname{det} \boldsymbol{D}=-\frac{1}{2} \operatorname{Tr}\left(\boldsymbol{D}^{2}\right) \\
& =-\frac{4 U^{2} a^{4}}{r^{6}}, \\
R_{\hat{t} \hat{\imath} \hat{\jmath}} & =0, \\
R_{\hat{\imath} \hat{\imath} \hat{t} \hat{\jmath}} & =-(\boldsymbol{v} \cdot \nabla) D_{i j}-\left(\boldsymbol{D}^{2}\right)_{i j} \\
& =-(\boldsymbol{v} \cdot \nabla) D_{i j}+\mathcal{P}_{i j} \operatorname{det} \boldsymbol{D},
\end{aligned}
$$

where the last line reads more explicitly

$$
\begin{aligned}
& R_{\hat{t} \hat{x} \hat{x} \hat{x}}=\frac{2 U^{2} a^{2}}{r^{8}}\left[a^{2}\left(y^{2}-5 x^{2}\right)+3\left(x^{4}-6 x^{2} y^{2}+y^{4}\right)\right], \\
& R_{\hat{t} \hat{y} \hat{t} \hat{y}}=\frac{2 U^{2} a^{2}}{r^{8}}\left[a^{2}\left(x^{2}-5 y^{2}\right)-3\left(x^{4}-6 x^{2} y^{2}+y^{4}\right)\right], \\
& R_{\hat{t} \hat{x} \hat{x} \hat{y}}=-\frac{12 U^{2} a^{2}}{r^{8}} x y\left(a^{2}-2 x^{2}+2 y^{2}\right) .
\end{aligned}
$$

These latter components show that the "circulation" $U a^{2}$ is not the only relevant parameter of the flow, in contrast to the constant-circulation vortex case, as we may expect from the reduced symmetry of the flow past the cylinder.

The curvature scalar

$$
R=\frac{8 U^{2} a^{4}}{r^{6}}
$$

decays much more quickly with distance from the cylindrical object than the curvature of the vortex flow, Eq. (58).

\section{E. Rigid rotation}

The simplest example of a nontrivial incompressible flow $(\operatorname{Tr} \boldsymbol{D}=0)$ is pure rotation $\boldsymbol{v}=\Omega(-y, x, 0)$, which has zero deformation $D_{i j}=0$, and constant vorticity $\omega_{z}=\omega_{0}=2 \Omega_{x y}=-2 \Omega_{y x}=2 \Omega$ (all other components vanishing). This flow is appropriate for instance deep inside the core of a vortex where the fluid effectively rotates as a "rigid" body. (In ordinary fluids this happens because viscosity dominates in the core; in superfluids there is a more dramatic effect in that the superfluid goes normal close enough to the core.) Also note that the core has a maximum size given by $|\boldsymbol{v}|=1$, that is $r_{c}=2 / \omega_{0}$.
For the rigid rotation flow it is easy to see that the Riemann curvature tensor is identically zero, either (1) by brute force application of the above formulae, or more subtly (2) by going to a rotating frame (of angular velocity $\left.\Omega=2 \omega_{0}\right)$ in which the velocity is identically zero, evaluating the Riemann tensor there (where it is blatantly zero), and transforming back to the rotating frame. Although the Riemann tensor is identically zero, there is interesting physics going on: The fact that pure rotation leads to zero Riemann curvature is ultimately responsible for the fact that equations (12) and (21) do not contain any terms quadratic in $\Omega$, a result that otherwise has to be simply asserted based on explicit calculation.

Additionally, we emphasise that even though the Riemann tensor is zero, the Christoffel symbols are definitely not zero. Indeed

$$
\begin{aligned}
\Gamma^{i}{ }_{t t} & =-\Omega^{2} r \hat{r}_{i}, \\
\Gamma_{t j}^{i} & =\Omega_{i j}=\frac{1}{2} \epsilon_{i j k} \omega^{k} .
\end{aligned}
$$

These two portions of the Christoffel symbols are of course simply representing the centrifugal and Coriolis pseudo-forces. All other components are zero.

A further (approximate) example of such a flow is encountered if one considers the coarse-grained flow induced by a lattice of vortices 25]. An (infinite) lattice rotates as if it were a solid body, with a vortex density $n_{v}=\Omega / \pi \gamma$ prescribed by the rotation velocity $\Omega$ and the circulation $2 \pi \gamma$, assumed to be equal for each individual vortex. For the vortex lattice, it follows from the vanishing of the Riemann curvature that a collimated quasiparticle beam can pass a (sufficiently dilute) lattice without (on average) being deflected.

\section{GEODESIC DEVIATION}

An invariant measure of the strength of a flow pattern as regards its influence on quasiparticle motion may be defined to be the value of the curvature scalar $R \propto s^{-\kappa}$ at a certain given distance $s$ from the flow-generating object (cf. Fig. 10 illustrating the generic situation of flow past an object placed in a homogeneous stream). Among the flows discussed in the previous section the shear flow is strongest in that sense (because the "flow generating object" is covering all space, $\kappa=0$ ), followed by the vortex flow $(\kappa=4)$ and the flow past the cylinder $(\kappa=6)$. Finally rigid rotation, which has zero $R$ and is "flat" $(\kappa=\infty)$. It is the simplest conceivable nontrivial (i.e., inhomogeneous) flow with the property of having all $R_{a b c d}$ equal to zero.

A nonvanishing Riemann tensor leads to tidal (relative) acceleration of nearby geodesics, described by the Jacobi equation of geodesic deviation for quasiparticles:

$$
\frac{D^{2} n^{\alpha}}{\mathrm{d} \lambda^{2}}+R_{\beta \gamma \delta}^{\alpha} u^{\beta} n^{\gamma} u^{\delta}=0 .
$$




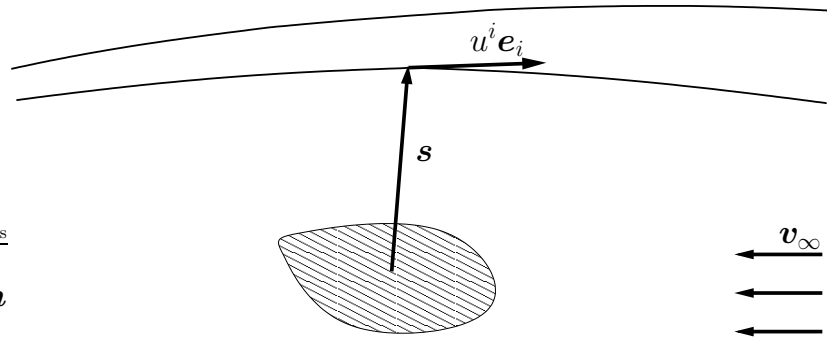

FIG. 1: The quasiparticle geodesic deviation at a distance vector $s$ caused by an object placed in a flow with velocity $\boldsymbol{v}_{\infty}$ at infinity (the generic case of the situation in section [IID).

The above relation gives the covariant relative acceleration of two nearby geodesics, with null tangent vectors $\boldsymbol{u}$ separated by the displacement vector $\boldsymbol{n}$, and with the geodesics affinely parametrized by $\lambda$. (At this stage all we need to know is that use of an "affine parameter" simplifies many formulae; in the following section we will derive a relationship between the affine parameter and physical Newtonian time $t$.) The fact that the constant time slices of the metric (5) are conformally identical to flat Cartesian space in three dimensions, entails that the spacetime curvature of the quasiparticle world is reflected in a relative acceleration of quasiparticle rays in the Newtonian lab world of nonrelativistic hydrodynamic flow.

Consider a family of geodesics in the $x$-direction, with tangent vector $\boldsymbol{u}^{a}=\left(u^{\hat{t}}, u^{\hat{t}}, 0,0\right)$ and a purely spacelike separation in the $y$-direction $\boldsymbol{n}=(0,0, \delta y, 0)$. We then have

$$
\frac{D^{2}[\delta y]}{\mathrm{d} \lambda^{2}}+\left\{\left(R_{\hat{y} \hat{t} \hat{y} \hat{t}}+R_{\hat{y} \hat{x} \hat{y} \hat{x}}\right)\left(u^{\hat{t}}\right)^{2}\right\}[\delta y]=0 .
$$

This can be viewed as a parametrically driven harmonic oscillator (driven in the affine parameter $\lambda$ ), with "frequency"

$$
\Omega(\lambda)=u^{\hat{t}} \sqrt{R_{\hat{y} \hat{y} \hat{y} \hat{t}}+R_{\hat{y} \hat{x} \hat{y} \hat{x}}}
$$

Physically this means that by looking at the components of the Riemann tensor we can see if the effective geometry locally acts as a focussing lens [corresponding to $\Omega(\lambda)$ real] or as a diverging lens [corresponding to $\Omega(\lambda)$ imaginary]. Since (in the focussing case, and assuming a reasonably uniform medium) two initially parallel geodesics will focus down to a point after an elapse of affine parameter $\delta \lambda=\pi / \Omega(\lambda)$, the corresponding local focal length is (in physical distance units) given by

$$
f^{\text {local }}=\frac{ \pm \pi}{\sqrt{\left\|R_{\hat{t} \hat{y} \hat{t} \hat{y}}+R_{\hat{x} \hat{y} \hat{x} \hat{y}}\right\|}} .
$$

Note the strengths and weaknesses of this concept it provides a local position and orientation dependent notion of focal length appropriate for nearly parallel geodesics (nearly parallel quasiparticles; so one is automatically working "on axis" and ignoring "spherical abberation"), but this definition of $f^{\text {local }}$ does in general not provide significant global information. If the Riemann tensor is strongly inhomogeneous, varying on length scales significantly smaller than $f^{\text {local}}$, then this concept of local focal length is not particularly useful. In particular, in the vortex geometry of [26], with flow (54), the focussing effect we had in mind was a global effect due to quasiparticles passing by opposite sides of the vortex core, with impact parameter $b$ - this is not a situation that can be described by the Jacobi equation. The global result obtained there for $f=f^{\text {global }}=$ $\left(2 b^{3} / 3 \pi r_{c}^{2}\right)\left[1+O\left(r_{c} / b\right)\right]$, is not the local $f^{\text {local }}$ defined above. Indeed two initially parallel quasiparticles passing by on the same side of the vortex core will be driven apart from each other by geodesic deviation - it is this effect that leads to the "cylindrical abberation" of the lens discussed in [26].

A case where the local focal length does acquire global meaning is the shear flow (50), for which the focal length (71) becomes a constant:

$$
f^{\text {shear }}=\frac{\sqrt{2} \pi}{\omega_{0}} .
$$

The focal length is in this case bounded by the atomic length scale itself, simply due to the requirement that the concept of hydrodynamics makes sense. This further strengthens the notion of the shear flow being the strongest possible flow as regards its influence on quasiparticle motion, because any other flow has more stringent bounds on the global $f$.

One useful refinement of the local focal length concept introduced in equation (71) is to consider null geodesics (quasiparticle paths) propagating in an arbitrary unit direction $\overline{\boldsymbol{u}}$ and then use indices $M$ and $N$ to denote the two spatial directions perpendicular to $\overline{\boldsymbol{u}}$. Then the local focal length can be generalized to a $2 \times 2$ matrix

$$
f_{M N}^{\text {local }}=\frac{ \pm \pi}{\sqrt{\left\|R_{\hat{t} M \hat{t} N}+R_{\hat{\imath} M \hat{\jmath} N} \bar{u}^{\hat{\imath}} \bar{u}^{\hat{\jmath}}\right\|}} .
$$

The square root and inverse is to be taken in the matrix sense, and the two eigenvalues of $f_{M N}$ are the two principal focal lengths along the direction $\overline{\boldsymbol{u}}$. If these eigenvalues differ it is a signal of astigmatism.

\section{NON-AFFINE PARAMETERIZATION OF NULL GEODESICS}

While the use of affine parameters for null geodesics is standard in general relativity, it should be borne in mind that in the present Painlevé-Gullstrand context there is a preferred temporal foliation provided by the Newtonian time parameter $t$. It is worth the technical bother of using the non-affine parameterization in terms of $t$ here in order to make aspects of the physics clearer. 
In general, we know that along any null geodesic there will be some relationship between affine parameter $\lambda$ and Newtonian time $t$. For instance we can assert

$$
\mathrm{d} \lambda=\exp [\zeta(t)] \mathrm{d} t
$$

In the affine parameterization the geodesic equation for a null curve is just

$$
u^{\mu} \nabla_{\mu} u^{\nu}=0 ; \quad u^{\nu} \equiv \frac{\mathrm{d} x^{\nu}}{\mathrm{d} \lambda} .
$$

If we choose a non-affine parameterization

$$
\bar{u}^{\mu} \nabla_{\mu} \bar{u}^{\nu}=\dot{\zeta}(t) \bar{u}^{\mu} ; \quad \bar{u}^{\nu} \equiv \frac{\mathrm{d} x^{\nu}}{\mathrm{d} t} .
$$

The geodesic equation becomes

$$
\frac{\mathrm{d}^{2} x^{\mu}}{\mathrm{d} t^{2}}=-\Gamma_{\alpha \beta}^{\mu} \frac{\mathrm{d} x^{\alpha}}{\mathrm{d} t} \frac{\mathrm{d} x^{\beta}}{\mathrm{d} t}+\dot{\zeta}(t) \frac{\mathrm{d} x^{\mu}}{\mathrm{d} t} .
$$

In this form it is clear that the physical acceleration of the quasiparticle is related to gradients in the PainlevéGullstrand metric. It is extremely useful to derive an explicit relationship between the affine parameter $\lambda$ and the physical Newtonian time $t$. To do this let's start with the notion of a stationary geometry (technically: there exists a timelike Killing vector; colloquially: a timeindependent geometry). The timelike Killing vector takes the form

$$
K^{\mu}=(1 ; \overrightarrow{0}) ; \quad K_{\mu}=\left(-\left[1-\boldsymbol{v}^{2}\right] ;-\boldsymbol{v}\right) .
$$

The tangent vector to the null geodesic is denoted

$$
u^{\mu}=\frac{\mathrm{d} x^{\mu}}{\mathrm{d} \lambda}=\frac{\mathrm{d} t}{\mathrm{~d} \lambda}\left(1 ; \frac{\mathrm{d} \boldsymbol{x}}{\mathrm{d} t}\right) .
$$

It is a standard theorem that the $3+1$ inner product between a geodesic tangent vector and a Killing vector is conserved, as long as the geodesic is affinely parameterized. Thus

$$
g_{\mu \nu} K^{\mu} u^{\nu}=\frac{\mathrm{d} t}{\mathrm{~d} \lambda}\left[1-\boldsymbol{v}^{2}+\boldsymbol{v} \cdot \frac{\mathrm{d} \boldsymbol{x}}{\mathrm{d} t}\right]=\text { constant. }
$$

On the other hand, because $u^{\mu}$ is a null vector

$$
1-\boldsymbol{v}^{2}+2 \boldsymbol{v} \cdot \frac{\mathrm{d} \boldsymbol{x}}{\mathrm{d} t}-\left|\frac{\mathrm{d} \boldsymbol{x}}{\mathrm{d} t}\right|^{2}=0 .
$$

Eliminating between these two equations, we can normalize in such a way that

$$
\frac{\mathrm{d} t}{\mathrm{~d} \lambda}=\exp [-\zeta(t)]=\left[1-\boldsymbol{v}^{2}+\left(\frac{\mathrm{d} \boldsymbol{x}}{\mathrm{d} t}\right)^{2}\right]^{-1} .
$$

That is

$$
\zeta(t)=\ln \left[1-\boldsymbol{v}^{2}+\left(\frac{\mathrm{d} \boldsymbol{x}}{\mathrm{d} t}\right)^{2}\right] .
$$

If the fluid is not moving, then $\boldsymbol{v}=0$ and $|\mathrm{d} \boldsymbol{x} / \mathrm{d} t|=$ 1 so $t \propto \lambda$. If the fluid is moving we simply have to live with this position-dependent factor relating the affine parameter $\lambda$ (in terms of which the geodesic equations are most easily written down) to the Newtonian time parameter $t$ (in terms of which the physical acceleration is most easily calculated).

In a similar manner, the Jacobi equation can be rewritten as

$$
\frac{D^{2} n^{\alpha}}{\mathrm{d} t^{2}}-\dot{\zeta}(t) \frac{D n^{\alpha}}{\mathrm{d} t}+R_{\beta \gamma \delta}^{\alpha} \bar{u}^{\beta} n^{\gamma} \bar{u}^{\delta}=0 .
$$

While this looks somewhat messier than the affinely parameterized Jacobi equation (68), the physics is the same. In particular if we start with two initially parallel null geodesics $(D n / \mathrm{d} t=0$ at $t=0)$, and assume a locally homogeneous medium, we are led to the same notion of local focal length as discussed in the previous section.

\section{DISCUSSION}

We have shown how the generation of curved Riemannian space-time geometries for quasiparticles is possible based purely on the velocity pattern of a nonrelativistic flow. Conversely, one might conceive of solving for a flow field from a given space-time geometry. This is a highly nonlinear problem, as becomes obvious from the relations (21) - (23). It is, however, certainly no more nonlinear or complicated than solving the Einstein equations of general relativity themselves. While the Painlevé-Gullstrand geometry discussed here does not provide us with the most generic case (remember that the constant time surfaces are (conformally) flat; for generalizations allowing for more general space-time metrics see [10]), it shows that the underlying kinematical structure of a curved space-time can in principle be perfectly nonrelativistic. The dynamical identification of this effective geometry with general relativity, i.e., imposing the Einstein equations, is a more advanced step [7], but is possible in principle as well.

There are several generalizations of the current analysis that would be of interest: (1) If the quasiparticle propagation speed ( $c$, local speed with respect to the background medium) is varying then the geometry exhibits "index gradient" effects in addition to effects generated by the motion of the medium. While technically straightforward, the relevant calculations of the Riemann tensor are computationally messy and the physical interpretation is not so clear (unless the medium is completely at rest; in which case one recovers standard "index gradient" physics). (2) If the density varies from place to place, then it is necessary to distinguish the "geometrical quasiparticle" regime (the analogue of geometrical optics) from the "wave quasiparticle regime" (the analogue of wave optics). In the geometrical approximation the results of the present paper can be carried over; in the wave regime one needs to carry out an analysis in 
terms of Green functions and wave equations; the entire armoury of quasiparticle trajectories as null geodesics of the effective metric breaks down and must be replaced by a more fundamental wave description.

In summary: The use of pseudo-Riemannian geometry has important applications well beyond the confines of general relativity. In particular quasiparticle propagation in condensed matter systems can often be characterized in terms of an "effective" spacetime geometry; most easily described in Painlevé-Gullstrand form. If the background medium is a fluid, then the Riemann curvature (and Christoffel symbols, etc.) can be calculated in terms of shear (deformation) and vorticity of the fluid. Ultimately this analysis relates the focussing and deflection of quasiparticles to the properties of the fluid flow.

\section{Acknowledgments}

URF acknowledges support by the Deutsche Forschungsgemeinschaft (FI 690/2-1) and the ESF Programme "Cosmology in the Laboratory". MV was supported by the US Department of Energy.
[1] E. Madelung: Quantentheorie in hydrodynamischer Form, Z. Phys. 40, 322 (1927).

[2] Sir Horace Lamb: Hydrodynamics, Republication of the sixth edition 1932, Dover, 1945.

[3] L. M. Milne-Thomson: Theoretical Hydrodynamics, Fifth Edition, Macmillan, 1968.

[4] W. G. Unruh: Experimental Black-Hole Evaporation?, Phys. Rev. Lett. 46, 1351 (1981).

[5] P. Painlevé: La mécanique classique et la théorie de la relativité, C. R. Hebd. Acad. Sci. (Paris) 173, 677-680 (1921);

A. Gullstrand: Allgemeine Lösung des statischen Einkörperproblems in der Einsteinschen Gravitationstheorie, Arkiv. Mat. Astron. Fys. 16, 1-15 (1922);

A generalization of the Painlevé-Gullstrand form of the Schwarzschild metric to the Kerr case may be found in C. Doran: New form of the Kerr solution, Phys. Rev. D 61, 067503 (2001);

A pedagogical discussion, in particular a comparison to Eddington-Finkelstein and Kruskal-Szekeres co-ordinates is contained in K. Martel, Eric Poisson: Regular coordinate systems for Schwarzschild and other spherical spacetimes, Am. J. Phys. 69, 476-480 (2001).

[6] M. Visser: Acoustic black holes: horizons, ergospheres, and Hawking radiation, Class. Quantum Grav. 15, 17671791 (1998);

M. Visser: Hawking radiation without black hole entropy, Phys. Rev. Lett. 80, 3436 (1998).

[7] G. E. Volovik: Superfluid analogies of cosmological phenomena, Phys. Rep. 351, 195-348 (2001).

[8] G. E. Volovik: Simulation of Painlevé-Gullstrand Black Hole in thin ${ }^{3} \mathrm{He}-\mathrm{A}$ film, JETP Lett. 69, 705-713 (1999) [Pis'ma Zh. Éksp. Teor. Fiz. 69, 662-668 (1999)].

[9] L. J. Garay, J. R. Anglin, J. I. Cirac, P. Zoller: Sonic analog of gravitational black holes in Bose-Einstein condensates, Phys. Rev. Lett. 85, 4643-4647 (2000);

L. J. Garay, J. R. Anglin, J. I. Cirac, P. Zoller: Sonic black holes in dilute Bose-Einstein condensates, gr-qc/0005131 Phys. Rev. A 63, 023611 (2001).

[10] C. Barceló, S. Liberati, M. Visser: Analogue gravity from Bose-Einstein condensates, Class. Quantum Grav. 18, 1137-1156 (2001).

[11] W. Gordon: Zur Lichtfortpflanzung nach der Relativitätstheorie, Ann. Phys. (Leipzig) 72, 421-456 (1923).

[12] U. Leonhardt: Space-time geometry of quantum dielectrics, Phys. Rev. A 62, 012111 (2000).
[13] R. Schützhold, G. Plunien, G. Soff: Dielectric Black Hole Analogs, quant-ph/0104121 Phys. Rev. Lett. 88, 061101 (2002).

[14] U. R. Fischer, G. E. Volovik: Thermal quasi-equilibrium states across Landau horizons in the effective gravity of superfluids, gr-qc/0003017 Int. J. Mod. Phys. D 10, 5788 (2001).

[15] Commonly the two notions of "quasiparticle" (pole in the Green function) and "elementary excitation" (giving energy vs. momentum curves) are used interchangeably in the literature, so also here. For a careful discussion of their differences see E. H. Lieb: Exact Analysis of an Interacting Bose Gas. II. The Excitation Spectrum, Phys. Rev. 130, 1616-1624 (1963).

[16] M. K. Parikh, F. Wilczek: Hawking Radiation As Tunneling, Phys. Rev. Lett. 85, 5042 (2000).

[17] R. Schützhold: On the Hawking effect, Phys. Rev. D 64, 024029 (2001).

[18] Georg Friedrich Bernhard Riemann: Über die Hypothesen, welche der Geometrie zu Grunde liegen, Springer, Berlin 1923, 3rd edition [Inaugural lecture, Göttingen, June 10th, 1854, with a commentary by H. Weyl].

[19] C. W. Misner, K. S. Thorne, J. A. Wheeler: Gravitation, Freeman, 1973.

[20] S. E. Perez Bergliaffa, K. Hibberd, M. Stone, M. Visser: Wave Equation for Sound in Fluids with Vorticity, cond-mat/0106255

[21] A. D. Pierce: Wave-equation for sound in fluids with unsteady inhomogeneous flow, J. Acoust. Soc. Am. 87, 2292-2299 (1990).

[22] M. Stone: Acoustic energy and momentum in a moving medium, cond-mat/9909315 Phys. Rev. E 62, 1341-1350 (2000).

[23] S. W. Hawking, G. F. R. Ellis: The Large Scale Structure of Space-Time, Cambridge University Press, 1973.

[24] A more general shear flow and its influence on quasiparticle geodesics in dielectric media has been considered by P. Ben-Abdallah: Propagation of light inside shear flows: a remote sensing method to retrieve velocity fields, J. Quant. Spectrosc. Rad. Trans. 73, 1-11 (2002).

[25] V. K. Tkachenko: On Vortex Lattices, Zh. Éksp. Teor. Fiz. 49, 1875 (1965) [Sov. Phys. JETP 22, 1282 (1966)].

[26] U. R. Fischer, M. Visser: Riemannian geometry of irrotational vortex acoustics, cond-mat/0110211 Phys. Rev. Lett. 88, 110201 (2002). 